\title{
New methods for determining the ages of PMS stars
}

\author{
Tim Naylor ${ }^{1}$, N.J. Mayne ${ }^{1}$, R.D. Jeffries ${ }^{2}$, S.P. Littlefair ${ }^{3}$ \& Eric S. \\ Saunders ${ }^{4}$ \\ ${ }^{1}$ School of Physics, University of Exeter Stocker Road, Exeter, EX4 4QL, UK. \\ ${ }^{2}$ Astrophysics Group, School of Physical and Geographical Sciences, Keele University, Keele, \\ Staffordshire ST5 5BG UK. \\ ${ }^{3}$ Department of Physics and Astronomy, University of Sheffield, Sheffield S3 7RH, UK. \\ ${ }^{4}$ Las Cumbres Observatory Global Telescope network, 6740 CortonaDrive, Suite 102, Goleta, \\ CA93117, USA
}

\begin{abstract}
We present three new methods for determining the age of groups of pre-mainsequence stars. The first, creating empirical isochrones allows us to create a robust age ordering, but not to derive actual ages. The second, using the width of the gap in colour-magnitude space between the pre-main-sequence and main-sequence (the radiative convective gap) has promise as a distance and extinction independent measure of age, but is as yet uncalibrated. Finally we discuss $\tau^{2}$ fitting of the main sequence as the stars approach the terminus of the main sequence. This method suggests that there is a factor two difference between these "nuclear" ages, and more conventional pre-main-sequence contraction ages.
\end{abstract}

Keywords. methods: statistical, methods: data analysis, stars: pre-main-sequence

\section{Introduction}

Good age determinations for pre-main-sequence (PMS) clusters and associations are crucial for our understanding of this phase of stellar evolution. For example, modelling the interaction of young stars with their (presumably) planet forming discs requires observational measurements of the disc dissipation and stellar spin-up timescales, both of which require accurate ages for the clusters and associations studied. Equally, any determination of the mass functions in young groups is strongly dependent on the assumed age. In this contribution we review three methods we are developing for measuring the ages of PMS clusters and associations using colour-magnitude diagrams (CMDs).

\section{Empirical isochrones}

Figure 1 shows CMDs for members of a selection of young clusters and associations. The majority of the stars lie on the PMS, which is elevated in the diagram with respect to the main sequence (MS). For older groups the stars lie closer to the MS, and this decline in luminosity with time is an age indicator. If the isochronal models for PMS stars were good fits to the data we could simply use the best-fitting ages. However, in practice the data deviate systematically from the isochrone (e.g. Bonatto 2004; Pinsonneault et al., 2004). Furthermore the derived ages can depend on which colour is fitted (e.g. Naylor et al., 2002). An obvious alternative is to abandon attempts to allocate absolute ages, but develop an age order (or ladder) based on the luminosity of the PMS. Simply plotting the data for different groups in the same diagram does not lead to useful results because the spread in each sequence is large. We therefore fit splines through each sequence, and place 


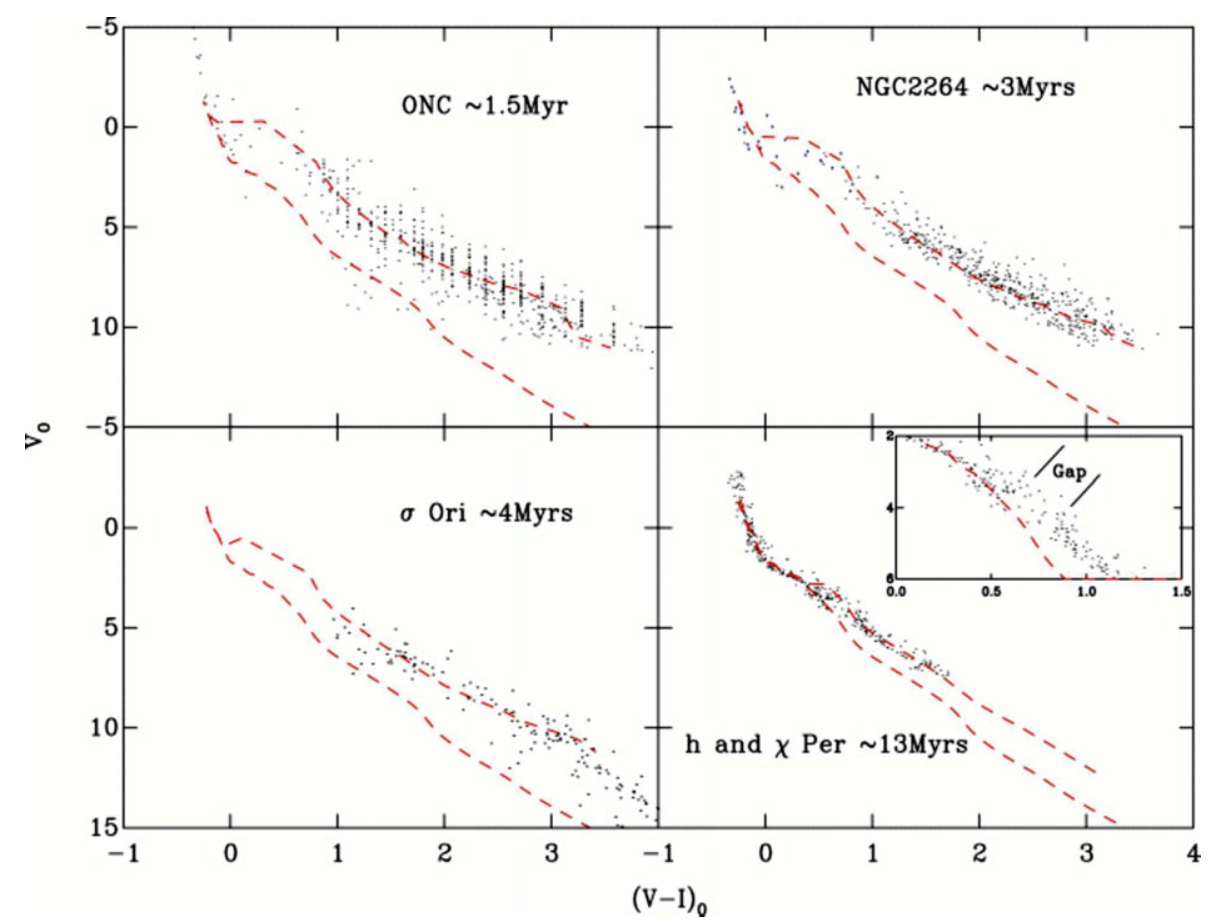

Figure 1. The CMDs for a selection of young groups in absolute magnitude and intrinsic colour. In each case the lower red dotted line is the position of the MS, the upper an appropriate Siess et al. (2000) isochrone.

Table 1. The Empirical Isochrone Age Ladder

\begin{tabular}{l|c}
\hline Age & Groups \\
\hline $1 \mathrm{Myr}$ & IC 5146 \\
$2 \mathrm{Myr}$ & ONC, NGC 6530 \\
$3 \mathrm{Myr}$ & $\lambda$ Ori, $\sigma$ Ori, NGC 2264 \\
$4-5 \mathrm{Myr}$ & IC 348, Cep OB3b ${ }^{1}$, NGC 2362 \\
$5-10 \mathrm{Myr}$ & $\gamma$ Vel $^{2}$ \\
$10 \mathrm{Myr}$ & NGC $7160^{2}$ \\
$13 \mathrm{Myr}$ & $\mathrm{a} d \& \chi$ Per \\
$40 \mathrm{Myr}$ & NGC 2547 \\
\hline
\end{tabular}

Notes: From Mayne \& Naylor (2008), except for: ${ }^{1}$ Littlefair (in prep); ${ }^{2}$ Jeffries et al. (2008)

the splines in absolute-magnitude, intrinsic-colour space. The result of such a procedure is shown in Figure 2 (left), where we can see that NGC2264 is older than the ONC, but younger than NGC1960 and about the same age as $\sigma$ Ori.

We used the above method in Mayne et al. (2007) to obtain an age order for a set of well known clusters and associations, but realised that the limiting factor was the determination of the distance (with which the age is degenerate). So, in Mayne \& Naylor (2008) we measured the distances to these clusters and associations in a consistent way. As can be seen in Figure 1 the most massive stars are actually on the MS. We therefore fitted these stars to a MS model using $\tau^{2}$ fitting (see Naylor \& Jeffries, 2006 and Section 4) to derive distances. In Table 1 we present the resulting age ordering, including two more associations from more recent work. Although we give ages in Table 1, it is worth emphasising that strictly speaking we only derive an order. The ages represent an informed average of literature PMS ages for these groups. 

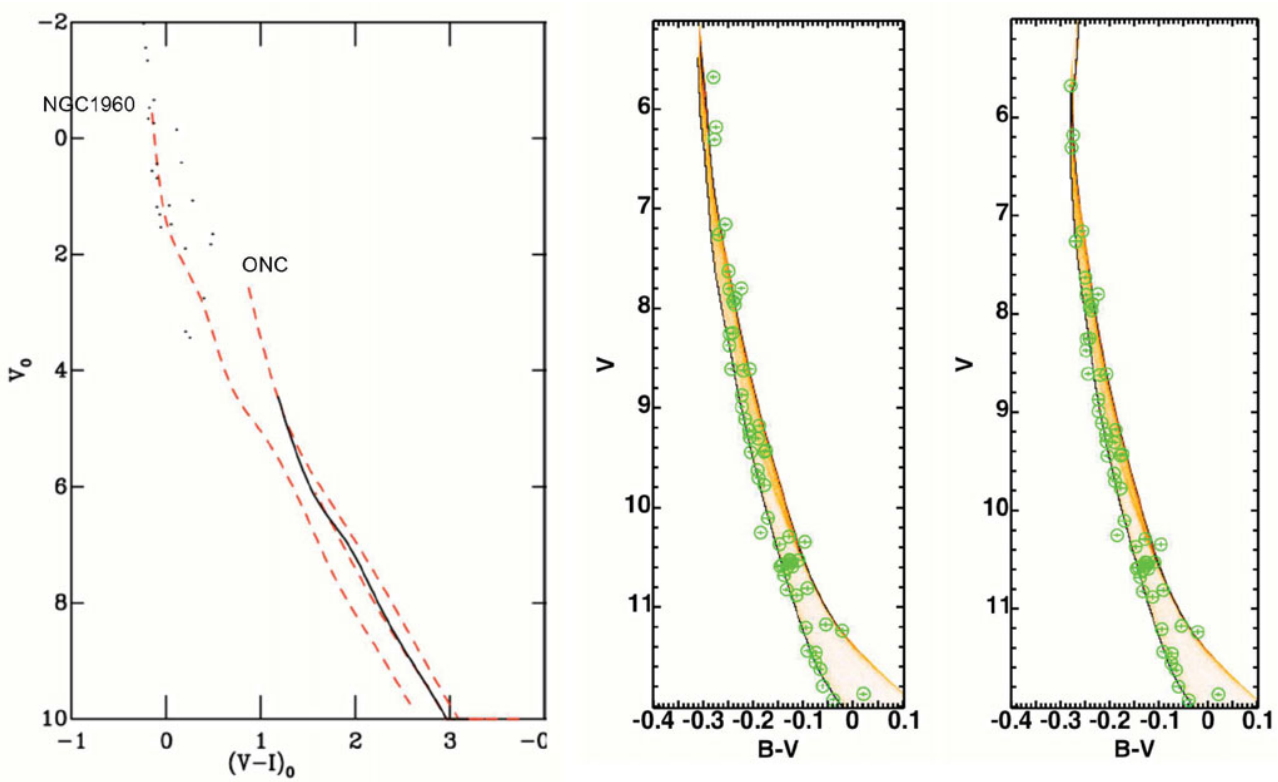

Figure 2. Left: The empirical isochrone for NGC2264 (black curve), compared with other clusters and associations (the red dashed curves). The unmarked red dashed isochrone close to that for NGC2264 is $\sigma$ Ori. Right: Geneva-Bessell isochrones fitted to the NGC6530 data of Walker (1957). Each star has been dereddened using its position in a $U-B / B-V$ diagram. The fit in the left hand panel had the age fixed at 0.25 Myr-old, yielding $\operatorname{Pr}\left(\tau^{2}\right)=0.03$. On the right the age was a free parameter, found to be $5.5 \mathrm{Myr}$, with $\operatorname{Pr}\left(\tau^{2}\right)=0.67$.

\section{Radiative-convective gap}

An interesting feature of Figure 1 is the paucity of stars on the PMS isochrone immediately prior to the point where it joins the MS. The gap is clear and wide for the youngest groups, but by the age of $h$ and $\chi$ Per has narrowed almost to the point of invisibility. The physical explanation for the gap lies in the change of structure between the fully convective interiors of PMS stars, and the partially radiative ones of MS stars. This drives a change in radius, which happens relatively quickly, leading to a rapid movement to bluer colours in the CMD, as stars move from the PMS to the MS. Hence in Mayne et al. (2007) we named this the radiative convective gap.

Clearly this change in the size of the gap with age could be used an age indicator. It has two main advantages over PMS (contraction) ages. First, it uses brighter stars. Second, since one is measuring a distance, rather than position on colour-magnitude space, it is independent of errors in distance or extinction (assuming the latter is uniform). Before it can be used as an age indicator, though, it will need to be calibrated against ages derived from other techniques.

\section{Nuclear (not quite turn-off) ages}

Before stars turn off the MS, they evolve redwards away from the zero-age MS, driven by their nuclear evolution. This means that in colour-magnitude space the MS, which normally has a positive gradient can, near its high-mass terminus, be vertical or even have a negative gradient (see Figure 2 (right)). Although the effect is subtle, if we can fit it, this should provide an age indicator. The best method for doing this is the $\tau^{2}$ method we described in Naylor \& Jeffries (2006), since this allows for the effects of 
binarity, gives reliable uncertainties for the parameters, and provides a goodness-of-fit test. Unfortunately the technique as we described it will not work if the isochrone is vertical. Therefore we first outline the improvements which we have made to the method to allow us to address this problem (which will be described in more detail in Naylor in prep.), before moving on to our results.

\subsection{Improvements to the $\tau^{2}$ technique}

The technique relies on a finely sampled grid (such as the colour scales of Figure 2 (right)), which is a model created from of order a million simulated stars. We refer to this as $\rho(c, m)$ where $c$ and $m$ are the colour and magnitude co-ordinates respectively. For any given datapoint we calculate $\tau^{2}$ by multiplying this grid on a point-by-point basis with a function representing the datapoint and its uncertainties (typically a twodimensional Gaussian). We represent this as $U_{i}\left(c-c_{i}, m-m_{i}\right)$, where $i$ is the index of a datapoint at co-ordinates $\left(c_{i}, m_{i}\right)$. If we now sum the resulting points, and repeat this over all datapoints we arrive at the definition of $\tau^{2}$

$$
\tau^{2}=-2 \sum_{i=1, N} \ln \int U_{i}\left(c-c_{i}, m-m_{i}\right) \rho(c, m) \mathrm{d} c \mathrm{~d} m .
$$

The best-fitting model corresponds to the lowest value of $\tau^{2}$. For example, a simple fit in distance modulus can be viewed as moving the models up and down in Figure 2 (right) until the "cross correlation" between the datapoints and the model is maximised. In practice the simplest way to find the best fitting model is to calculate $\tau^{2}$ for a grid of models covering the range of parameters of interest.

There is a question as to how $\rho$ should be normalised. In Naylor \& Jeffries (2006) we derived a normalisation such that Equation 4.1 reduced to that for $\chi^{2}$ for fitting a curve to data with uncertainties in one dimension. Unfortunately, when the isochrone is vertical, this results in an infinity in Equation 17 of that paper making it impractical for post-main-sequence fitting. Instead we now use a normalisation where the integral of $\rho$ between the faintest and brightest datapoints is one. Similarly we demand that the integral of $U$ is one over the entire CMD.

Having found a fit to the data, we must establish whether it is a good fit. We do this by calculating the probability that we would obtain our value of $\tau^{2}$ from observations, assuming the model was correct. This is $\operatorname{Pr}\left(\tau^{2}\right)$, which we showed how to calculate for no free parameters in Naylor \& Jeffries (2006). Our suggested correction to allow for free parameters, multiplying the values of $\tau^{2}$ by $(N-n) / N$ (where $N$ is the number of datapoints and $n$ the number of free parameters), is not invariant under changes in normalisation. A better approximation is to subtract the expectation value of $\tau^{2}$ before multiplying by $(N-n) / N$, and then add the expectation value on again.

Finally, to find the uncertainties in the parameters we have found a quicker method than that we presented in Naylor \& Jeffries (2006). Assuming the minimum value of $\tau^{2}$ has been found by a grid search, each datapoint in the grid has a probability $P$ associated with it (via the definition of $\tau^{2}$ ) of

$$
P=e^{-\tau^{2} / 2}
$$

By summing the probability below a given $\tau^{2}$, and dividing by the probability summed over the entire grid, one can obtain the probability that $\tau^{2}$ lies below a given value. This allows one to draw a confidence contour in the parameter space, in an identical fashion to that used in $\chi^{2}$ analysis. 


\subsection{Results}

We have fitted UBV photometry for bright stars in NGC6530, NGC2264, $\sigma$ Ori, $\lambda$ Ori, NGC2362, Cep OB3b, NGC2547, IC2602 and stars in the vicinity of the Orion Nebula Cluster. For preference we have used data from the 1950s to 1970s of Walker, Johnson and collaborators, since this is a relatively homogeneous group of datasets, and we find our models fit them well. We have used the Geneva-Bessell models described in Mayne \& Naylor (2008). We first use a $U-B / B-V$ diagram to determine the extinction. In some cases the extinction is uniform, and we determine its value using $\tau^{2}$ fitting. In other cases we find the extinction is non-uniform, and we find the extinctions on a star-by-star basis by comparison with the isochrone as described in Mayne \& Naylor (2008). This is essentially an updated $Q$ method. We then perform a grid search in both age and distance to derive ages and associated uncertainties. Unsurprisingly our distances are all consistent with those in Mayne \& Naylor (2008), but for the groups less than 10 Myr old, we find the ages are a factor 1.5-2.0 larger than those given in Table 1.

Before attaching any significance to this result, we questioned whether it could be due to either our fitting procedure or the models used. We have experimented with models without convective overshoot, which we find give poor fits to the data, and with the Padova models (Girardi et al. 2002), which we find give similar answers to the GenevaBessell isochrones, though both models have yet to be tested exhaustively. We also find that our age for stars in the vicinity of the ONC (5 Myr) is similar to that found using the same dataset by Meynet et al. (1993). For IC2602 Mermilliod (1981) obtains a nuclear age of $35 \mathrm{Myr}$, which compares favourably with our estimate of about $40 \mathrm{Myr}$. For NGC 2547 we obtain about $45 \mathrm{Myr}$, compared with Claria (1982) who obtains $57 \mathrm{Myr}$. Since our ages are broadly consistent with other turn-off/nuclear ages, we can rule out some systematic effect from our models or fitting procedure. We are, therefore, forced to conclude that this is a genuine discrepancy between PMS ages and nuclear ages.

For the groups older than $10 \mathrm{Myr}$ it is harder to be definitive about any difference. The problem is we need both PMS photometry (to obtain an age on the same scale as Table 1) and good photo-electric photometry to obtain a nuclear age. We can carry out the test for NGC2547 for which we obtain $45 \mathrm{Myr}$, compared with PMS and Lithium depletion ages of about $38 \mathrm{Myr}$ (Naylor \& Jeffries, 2006) suggesting the discrepancy decreases with age. For IC2602 the situation is more ambiguous. The age we obtain (40 Myr) is larger than the PMS age of $25 \mathrm{Myr}$ found by Stauffer et al. (1997), but is consistent with the finding of Jeffries et al. (2000) that IC2602 is a little older than NGC2547. Thus the question as to whether this discrepancy disappears for older clusters (and if so at what age) must await obtaining further PMS ages for older clusters.

\subsection{Implications}

It appears we have found a genuine difference between the PMS contraction and MS nuclear age scales. It is hard at this stage to decide which scale is correct, but as most modern work relies on the PMS age scale, it is interesting to examine the implications of the nuclear age scale being correct. It would help address two outstanding problems in the area. First, it is well known that that there appears to be a lack of clusters in the age range 5-30 Myr (Jeffries et al., 2007). Changing the ages in the way we suggest would fill that gap, especially if there is a return to the classical age scale for clusters older than $30 \mathrm{Myr}$. Second there is a problem in the disc-clearing timescale measured from IR observations of young stars (of order $3 \mathrm{Myr}$, e.g. Briceño et al., 2007) and the time required to form a planet by classical core accretion (perhaps $9 \mathrm{Myr}$; Pollack et al., 1996). Whilst there are active attempts to solve this problem by reducing the theoretical 


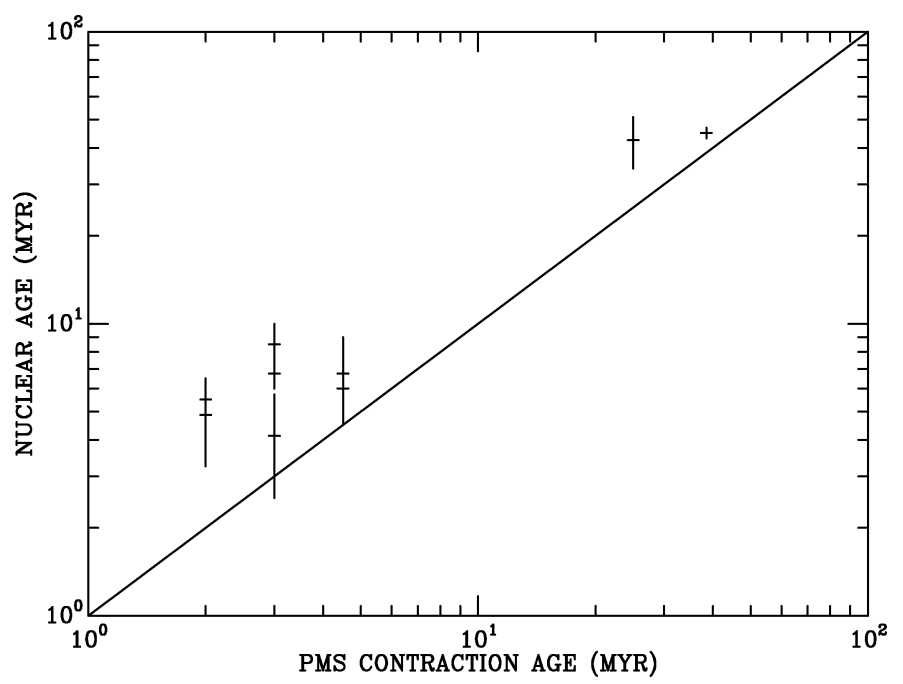

Figure 3. PMS vs nuclear ages for young clusters and associations. The datapoints would lie on the line if they were equal. The error bars show the uncertainties derived from the $\tau^{2}$ fitting.

timescale (e.g. Dodson-Robinson et al., 2008 and references therein), our work supports a different solution - increasing the observed timescale by a factor two.

\section{References}

Bonatto, C., Bica, E., \& Girardi, L. 2004, A\&A 415, 571

Briceño, C., Preibisch, T., Sherry, W. H., Mamajek, E. A., Mathieu, R. D., Walter, F. M., \& Zinnecker, H., in proceedings of Protostars and Planets $V \mathrm{p} 345$

Claria, J. J., 1982, A\&AS 47, 323

Dodson-Robinson, S. E., Bodenheimer, P., Laughlin, G., Willacy, K., Turner, N. J., \& Beichman, C. A., 2008, 2008arXiv0810.0288D

Girardi, L., Bertelli, G., Bressan, A., Chiosi, C., Groenewegen, M. A. T., Marigo, P., Salasnich, B., \& Weiss, A. 2002, A\& $A$ 391, 195

Jeffries, R. D., Totten, E. J., \& James, D. J., 2000, MNRAS 316, 950

Jeffries, R. D., Oliveira, J. M., Naylor, T., Mayne, N. J., \& Littlefair, S. P. 2007, MNRAS 376, 580

Jeffries, R.D., Naylor, T., Walter, F. M., Pozzo, M. P., \& Devey, C. R., 2008, MNRAS, accepted, arXiv:0810.5320

Mayne, N. J. \& Naylor, T., 2008, MNRAS 386, 261

Mayne, N. J.,Naylor, T., Littlefair, S. P., Saunders, E. S., \& Jeffries, R. D., 2007, MNRAS 375, 1220

Mermilliod, J. C., 1981, A\& A 97, 235

Meynet, G., Mermilliod, J.-C., \& Maeder, A., 1993, A\&AS 98, 477

Naylor, T., Totten, E. J., Jeffries, R. D., Pozzo, M., Devey, C. R., \& Thompson, S. A. 2002, MNRAS 335, 291

Naylor, T. \& Jeffries, R. D. 2006, MNRAS 373, 1251

Pinsonneault, M. H., Terndrup, D. M., Hanson, R. B., Stauffer, J. R. 2004, ApJ 600, 946

Pollack, J. B., Hubickyj, O., Bodenheimer, P., Lissauer, J. J., Podolak, M., \& Greenzweig, Y., Icarus 124,62

Siess, L., Dufour, E., \& Forestini, M. 2000, ApJ 358, 593

Stauffer, J. R., Hartmann, L. W., Prosser, C. F., Randich, S., Balachandran, S., Patten, B. M., Simon, T., \& Giampapa, M., 1997, ApJ 479, 776

Walker, M. F. 1957, ApJ 125, 636 


\section{Discussion}

E. MAMAJEK: Your ages for high masses are critically dependent on converting the reddening-free $Q$-parameter and de-reddening the stellar photometry. There are issues with inferring $\mathrm{T}_{\text {eff }}$ from $Q$ and $(B-V)_{0}$, i.e., disagreements among various studies. There are also uncertainties in the calibration of $\mathrm{T}_{\text {eff }}$ for OB stars. Could you discuss how these uncertainties affect the certainty of your conclusions that the pre-MS ages are a bigger problem than the ages of the high mass stars?

T. NAYlor: So far I have only tested the Padova and Geneva isochrones. They agree which is more than any two PMS isochrones do! If the extinction were a problem I would expect to see a correlation with $A_{V}$, which I don't.

F. WALter: Just to clarify, is this an age offset or a stretching of the PMS time-scale?

T. NAYLOR: At this point it is hard to say whether it's an offset, say 4 Myr or a multiplicative factor which decays to zero by, say, $10 \mathrm{Myr}$. The statistics are just too poor. 


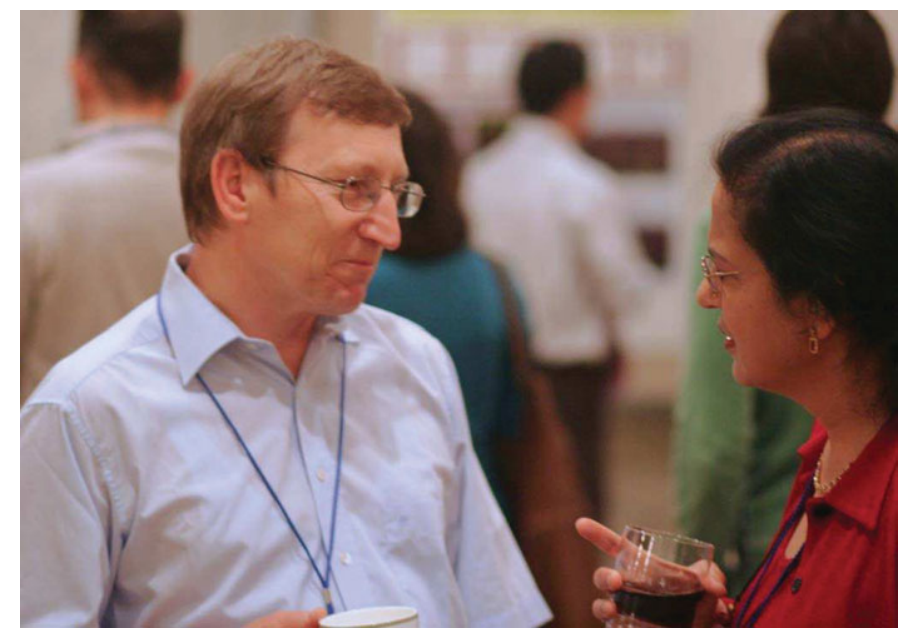

Janusz Kaluzny and Hashima Hasan

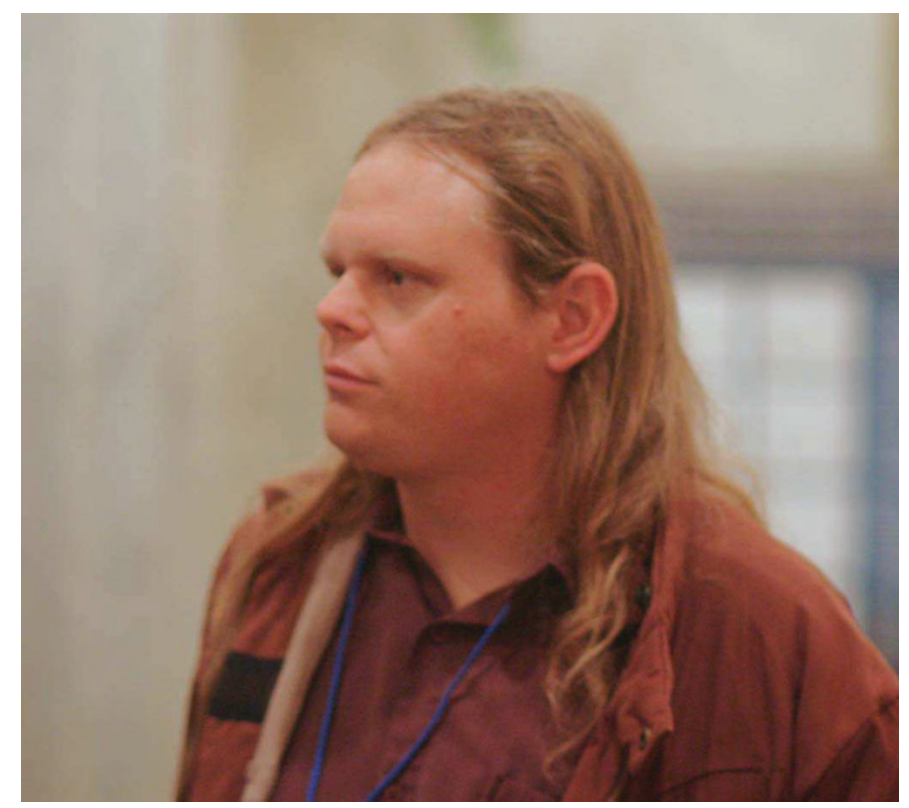

Patrick Young 